\title{
Comments on UM: Universal Morphology by Michal Starke, NELS 2020-21
}

\author{
Hagit Borer \\ Queen Mary University of London \\ h.borer@qmul.ac.uk
}

How to cite: Borer, Hagit. 2021. Comments on UM: Universal Morphology by Michal Starke, NELS 2020-21. Isogloss. Open Journal of Romance Linguistics 7, 14: 1-4.

Michal Starke's (MS) presentation is a very impressive take on deriving the phonological realization of the French verbal paradigm, introducing systematicity into the inflectional system where previous accounts appear to have failed, and signaling, or so it might be hoped, the potential availability of a principled treatment for the notorious morpho-phonological irregularity of inflectional paradigms. I would not indeed be surprised if the system can be successfully extended to other synthetic or semi synthetic languages (but see caveats below). ${ }^{1}$

I do have an important theoretical query, however, the answer to which is fundamental to the overall assessment of the account and to its placement within our overall view of the grammar. The system crucially relies on lexical entries to 'check' the emerging syntax, with syntactic structural fragments compared, at fixed junctures, with the availability of suitable lexicalizations. Cases of movement ('movement to lexicalize') in turn are precisely cases where there are mismatches between the lexical entry and the syntax, with the movement aiming at matching the lexical entry. It therefore emerges that the nature of lexical entries and how they are constructed is

\footnotetext{
Not being a Romance specialist, I will assume unless shown otherwise that the system is empirically solid.
} 
critical to the assessment of the system. Equally crucial is a full articulation of the mapping of lexical structures to phonological representations.

The lexical representations, as given, are complex, labeled hierarchical structural fragments which are built from the root up. Their phrasal level ('XP') needs to match that of the syntactic phrase they are matched with, and to a large extent, their hierarchical structure mirrors that of syntactic representations. The match, however, is not perfect, with cases of mismatches, as noted, motivating movement. However, the presentation as it now stands does not address the principles which govern the construction of the hierarchy within lexical entries, and in particular, the reason for the considerable overlap with syntactic hierarchies on the one hand, alongside the possibility of 'deviations' from the syntactic hierarchy, on the other hand.

Lacking an elaboration on the way in which lexical structures/entries are constructed, some overall important issues cannot be assessed. For instance, the existence of two hierarchical representations which largely (but not fully) overlap raises the question of whether both are needed, as another possibility would be that what we have here is a model of lexical actualization which is independent of (narrow) syntax, into which the output of such structure is inserted as a terminal. This possibility can be neither pursued nor excluded, however, in the absence of a better understanding of how lexical entries are constructed and constrained.

Equally unresolved, in the presentation, is the matter of the mapping between the incremental lexical structural fragments and phonological representations, a matter that can be illustrated by considering the properties of Semitic verbs. The Semitic verb, although structurally and phonologically highly predictable, has nonetheless been a considerable challenge for representational systems, including potentially the present one, where lexical entries are, by assumption, representations. The challenge goes well beyond the presence of prefixes or possibly infixes (as acknowledged in the discussion session), and consists of at least two characteristics: the first is the existence of intermediate (syntactic) derivational stages with a partial structure which does not, and indeed cannot constitute a well-formed phonological word. The second characteristic involves discontinuous morphemes. A (simplified) illustration of the 'partial structure' issue from Hebrew is in the structures in (1)-(3): ${ }^{2}$

2 While accounts of the functional structure associated with the Semitic verbal template are not always agreed upon, present-day accounts all share the assumption that the derivation of the Semitic verb involves intermediate derivational stages at which the representation cannot, in and of itself, be phonologically well-formed, as it excludes the vocalic representation. The representation in (1) is along traditional historical treatments as articulated, within contemporary grammatical models, in Arad (2005) and modified in Borer (2013, 2021). For a different functional approach see Doron (2003) and Kastner (2016). As is to be expected, the very same issues face residual 'Semitic' morphology in allomorphic cases such as English sing/sang/sung, or German syntactically conditioned vocalic stem changes. 


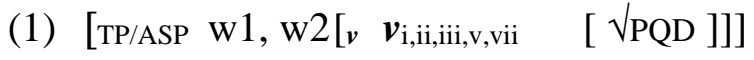

(2) a. [TP/ASP w1, w2 [ $\left.\begin{array}{llll}v & \mathrm{PQD}\left(\boldsymbol{v}_{\mathrm{i}}\right) & \ldots & \langle\sqrt{\mathrm{PQD}}\rangle\end{array}\right]$

b. [TP/ASP w1, w2 [ $\left.\begin{array}{llll}v & n i \mathrm{PQD}\left(v_{\mathrm{ii}}\right) & \langle\sqrt{\mathrm{PQD}}>\end{array}\right]$

'(give) order'

c. [TP/ASP w1, w2 [v PQQD $\left.\left(v_{\text {iii }}\right) \quad\langle\sqrt{ } \mathrm{PQD}>]\right]$

'be absent'

d. [TP/ASP w1, w2 [v $\left.h \begin{array}{lll}v & h \mathrm{PQD}\left(v_{\mathrm{v}}\right) & \langle\sqrt{\mathrm{PQD}}>\end{array}\right]$

'command'

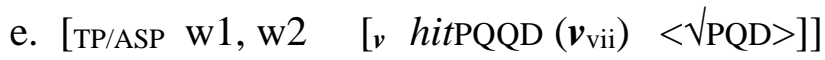

'deposit, entrust'

'be counted'

(3)

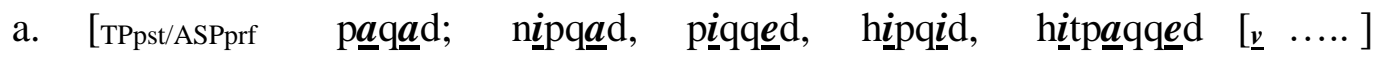

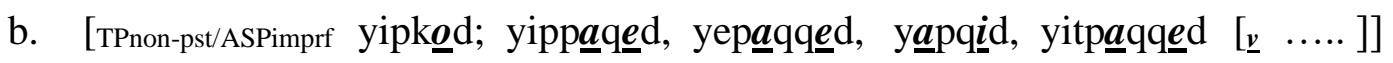

in (1), vi,ii,iii,v, vii stand for five different possible prefixal/infixal exponents of a verbal categorial label, $v$ (templates, binyanim, keys). The combination of the root with any exponent of $\mathrm{v}$ gives rise to a form which is not a licit phonological word (but which is associated with fixed listed meaning, see Borer, 2021). The emergence of a well-formed phonological word, in turn, is contingent on typically 2 vowels associated with tense (or aspect) which, in Hebrew, differ from one $\mathrm{v}$ exponent to the next as well as from past/perfect and non-past/imperfect, (and which, in themselves, are realized discontinuously. Notated as $\mathrm{w} 1, \mathrm{w} 2$ in (1-2), bold italics in the full representations in (3).

Within output-output phonological systems as well as within lexeme-based paradigmatic approaches, the absence of an attested, or even possible phonological word corresponding to partial derivational stages has led to a wholesale denial of the existence of Semitic root/template morphology, and its replacement with a set of adjustment rules or melodic over-writing which operate on listed fully formed words/lexemese. That, in spite of the fact that the Semitic verb is extremely easy to segment and decompose and with the exception of the first template $\left(\mathrm{v}_{\mathrm{i}}\right)$, is marked by near-complete predictability. ${ }^{3}$ To the extent that the system proposed by MS crucially involves lexical representations, one wonders how partial representations such as those in (1), in particular, would be treated, given the fact that they are not possible phonological words?

A related conceptual issue which presents a prima facie challenge to representational systems, and a particular challenge to the bottom-up lexical representations proposed by MS concerns discontinuous representations such as those in (3), in which the root radicals are interspersed with the vocalic tense/aspect vocalic markings. Under the assumption that the syntactic tree and the lexical tree have the same constituent structure, it is not easy to see, without better understanding of how lexicalization work, how the lexicalization of $[v$ PQD $(v)<\sqrt{ } \mathrm{PQD}>]$ as given in (1)

3 'Irregularity', for the exponents $\boldsymbol{v}_{\mathrm{i}, \mathrm{iii}, \mathrm{v}, \mathrm{vii}}$ consists exclusively of predictable phonological allomorphy triggered by the phonological properties of glides or pharyngeal root radicals. This said, Modern Hebrew pronunciation has lost some of the phonological distinctions which trigger such phonological allomorphy (e.g., the distinction between ? and ९), , but has kept the allomorphy for the relevant roots, thereby giving rise to a higher degree of apparent irregularity. 
could give rise to the lexicalizations in (3), under the assumption, of course, that we do want to avoid the arbitrary independent listing of the set of all possible outputs. Derivational approaches which seek to avoid melodic over-writing typically handle the issue by postulating an abstract prf/imperf morpheme and a phonological linearization procedure, but the degree to which such solution is available within the model currently under consideration is not clear.

I have pointed out several areas where the evaluation of MS's proposal is impossible without a more articulated understanding of the properties of lexical items. Some of these involve potential challenges both to the incremental nature of the lexicalizations proposed, and to the bottom-up process of syntax/lexicon matching. The others involve the inability to fully assess what, exactly, the proposed model is a model of (Syntax and Lexicon? Just Lexicon?) without a better understanding of the relationship between syntactic and lexical hierarchy building operations. The 'inflectional' model, as it stands, is attractive enough, however, to make the pursuit of further elaboration worthwhile, in the hope that appropriate answers could be provided.

\section{References}

Arad, Maya. 2005. Roots and Patterns: Hebrew Morphosyntax. Dordrecht: Springer.

Borer, Hagit. 2013. Taking Form, Structuring Sense Volume III. Oxford: Oxford University Press.

Borer, Hagit. 2021. Getting to the Root. Paper presented at Grammar Architecture Morphology and Its Interfaces, Abralin.

https://aovivo.abralin.org/lives/grammar-architecture-2/

Doron, Edit. 2003. Agency and voice: the semantics of the Semitic templates. Natural Language Semantics 11:1-67.

Kastner, Itamar. 2016. Form and Meaning in the Hebrew Verb. PhD dissertation, NYU. 